\title{
Prevalence and risk factors of active pulmonary tuberculosis among elderly people in China: a population based cross- sectional study
}

Can-You Zhang ${ }^{1}$, Fei Zhao', Yin-Yin Xia', Yan-Ling Yu², Xin Shen ${ }^{3}$, Wei Lu' ${ }^{4}$, Xiao-Meng Wang ${ }^{5}$, Jin Xing ${ }^{6}$, Jian-Jun Ye ${ }^{7}$, Jian-Wei Li $i^{8}$, Fei-Ying Liu' ${ }^{9}$ Jian-Lin Wu ${ }^{10}$, Lin Xu ${ }^{11}$, Hui Zhang ${ }^{1 *}$, Jun Cheng ${ }^{1 *}$ and Li-Xia Wang ${ }^{1 *}$

\begin{abstract}
Background: The problem of population aging is a critical public health concern in modern China, and more tuberculosis (TB) control efforts are needed to reach elderly people at high priority. In this study, we aim to determine the prevalence and identify the risk factors of TB among elderly people in China.
\end{abstract}

Methods: A multistage cluster-sampled cross-sectional survey was conducted in 2013, and 27 clusters were selected from 10 counties of 10 provinces in China. All consenting participants greater than or equal to 65 years of age were screened for pulmonary TB with a chest X-ray (CXR) and a symptom questionnaire. Three sputum specimens for bacteriological examination by microscopy and culture were collected from those whose screening was positive. Prevalence was calculated, a multiple logistic regression model was performed to confirm the risk factors, and population attributable fraction (PAF) of each risk factor was calculated to indicate the public health significance.

Results: Of 38888 eligible people from 27 clusters, 34269 participants finished both questionnaire and physical examination. There were 193 active pulmonary TB cases, 62 of which were bacteriologically confirmed. The estimated prevalence of active pulmonary TB and bacteriologically confirmed TB in those 65 years of age and older was 563.19 per 100000 (95\% Cl: 483.73-642.65) and 180.92 per 100000 (95\% Cl: 135.89-225.96), respectively. Male sex, older age, living in rural areas, underweight, diabetes, close contact of pulmonary TB (PTB) and previous TB history are all risk factors for TB. The risk of TB increased with increasing age and decreasing body mass index (BMI) after adjusting for other factors, and there is a positive dose-response relationship.

Conclusions: In China, active case finding (ACF) could be implemented among elderly people aged 65 and above with underweight, diabetes, close contact history and previous TB history as a priority, which will get significant yields and be cost-effective.

Keywords: Prevalence, Risk factor, Pulmonary tuberculosis, Aged/elderly

\footnotetext{
* Correspondence: zhanghui@chinacdc.cnl; chengjun@chinacdc.cn; wanglx@chinacdc.cn

${ }^{1}$ National Center for Tuberculosis Control and Prevention, Chinese Center for

Disease Control and Prevention, Beijing, China

Full list of author information is available at the end of the article
}

(c) The Author(s). 2019 Open Access This article is distributed under the terms of the Creative Commons Attribution 4.0 International License (http://creativecommons.org/licenses/by/4.0/), which permits unrestricted use, distribution, and reproduction in any medium, provided you give appropriate credit to the original author(s) and the source, provide a link to the Creative Commons license, and indicate if changes were made. The Creative Commons Public Domain Dedication waiver (http://creativecommons.org/publicdomain/zero/1.0/) applies to the data made available in this article, unless otherwise stated. 


\section{Multilingual abstracts}

Please see Additional file 1 for translations of the abstract into the five official working languages of the United Nations.

\section{Background}

Population aging is a critical public health concern in modern China. The proportion of elderly people aged 65 and above was $10.8 \%$, and people aged 60 and above $16.7 \%$ of a population of 1382 million people in 2016 [1]. The risk of tuberculosis (TB) increases with age, as shown by studies internationally [2-5] and in China [6]. Almost half (48.8\%) of the diagnosed TB cases in China's Fifth National Prevalence Survey of TB were elderly people, among whom $39.8 \%$ were asymptomatic, and $53.2 \%$ didn't seek any medical care [7]. Bele et al. found that population aging was one of the bottlenecks in TB control in rural China, and more TB control efforts are needed to reach the most vulnerable populations at high priority [8].

Rapid case identification and early treatment are the most important interventions to prevent TB transmission and reduce its incidence $[9,10]$. Systematic screening of high-risk groups to diagnose TB early has proven effective to help end the global TB epidemic [11, 12]. However, it's not feasible to screen all elderly people due to the huge population numbers in China. On the other hand, combining age with one or more TB risk factors could detect a higher TB prevalence in a more concentrated population. In this study, we aim to determine the prevalence and identify the risk factors of TB among elderly people to shape the TB screening algorithm for this high-risk population in China.

\section{Methods}

\section{Study setting and sampling}

This was a cross-sectional study. Sample size was estimated using a method appropriate to estimate a single population proportion. The $369 / 100000$ prevalence of bacteriologically positive pulmonary TB (PTB) among elderly people ( $\geq 65$ years) from the most recent national TB prevalence survey was used as the estimated prevalence of elderly population (unpublished data). We assumed 95\% confidence interval and 0.2 allowable error and used the formula $n=\frac{p q}{\left(\frac{d}{z_{\alpha}}\right)^{2}}(p=369 / 100000, \mathrm{q}=1-$ $\left.\mathrm{p}, \mathrm{d}=0.25 \mathrm{p}, \alpha=0.05, Z_{\alpha}=1.96\right)$. A design effect of 1.8 from the simple random sampling was considered. So, a total of 29873 elderly participants were needed and a sample size of 33192 was determined to allow $10 \%$ of non-response.

We applied the multistage sampling in 27 study sites (10 townships in rural areas and 17 communities in urban areas) from ten counties of ten provinces in
China. In the first stage, ten out of 31 provinces were selected, of which three were from eastern China, three from central China, three from western China, and one from four municipalities directly under the central government (Beijing, Shanghai, Tianjin and Chongqing). Selection at this stage was also based on willingness to participate and the human resources of each province. In the end, Sichuan, Guangxi, Yunnan of western China, Heilongjiang, Henan, Hubei of central China, Zhejiang, Jiangsu, Guangdong of eastern China, and Shanghai were chosen. In the second stage, one county per district that had more than 500000 people was randomly selected in each province. In the third stage, random cluster sampling was used to select communities or townships. If the total number of general population in selected community or township was less than 30000 , then the nearest community or township would also be included in the study site, to reach 30000 . Finally, a total of 38888 elderly people were eligible participants in the study.

\section{Definitions}

Suspected TB symptoms were defined by this study, as any of the following conditions: (1) cough for more than 2 weeks; (2) hemoptysis; (3) cough for more than 1 week yet less than 2 weeks, and accompanied with any of the following symptoms: fever, chest pain, night sweating, loss of appetite, fatigue, and/or weight loss (>3 kg). The definitions of previous TB cases, human immunodeficiency virus or acquired immune deficiency syndrome (HIV/AIDS), known diabetes, close contacts, underweight, tobacco use, drinking history, chronic bronchitis, average family annual income per capita and average family living area, are shown in Table 1. It is necessary to note that China has its own criteria of body mass index (BMI) for adults: underweight $\mathrm{BMI}<18.5$, normal $18.5 \leq \mathrm{BMI}<23.9$, overweight $\mathrm{BMI} \geq 24$ [13].

\section{Data collection}

In our study, each province organized a research team of 50-100 staff for data collection, including researchers, health-care workers, enumerators, and local government staff. From June to September 2013, participants were interviewed for any suspected TB symptoms. Meanwhile, information of participants' sex, age, marital status, education, medical history, smoking and drinking habit, and socioeconomic status were collected. Their height and weight were measured to calculate the BMI as an indicator of nutritional status. All participants were offered chest X-ray (CXR) examination. The interviews took approximately 15-20 min each CXR was completed in less than $1 \mathrm{~h}$ including waiting time for one participant.

Participants with any one of the suspected TB symptoms or CXR abnormalities consistent with TB were asked to submit three sputum samples (morning, night 
Table 1 Definitions of terms used in this study

\begin{tabular}{|c|c|}
\hline Term & Definition \\
\hline Previous TB cases & Registered in TB Management Information System, and finished treatment or cured. \\
\hline HIV/AIDS & $\begin{array}{l}\text { Registered in local CDC database, whom were diagnosed according to diagnostic criteria for HIV/AIDS } \\
\text { published by National Health Commission of the People's Republic of China in } 2008 .\end{array}$ \\
\hline Known Diabetes & $\begin{array}{l}\text { Recorded on the Citizen Health Management Files as diagnosed with Diabetes (fasting plasma glucose level } \\
\geq 7.0 \mathrm{mmol} / \mathrm{L} \text {, or } 2 \text {-h plasma glucose level } \geq 11.1 \mathrm{mmol} / \mathrm{L} \text { ), plus those using medicine to control blood glucose } \\
\text { by self-report. }\end{array}$ \\
\hline Close Contacts & Living with new active PTB case for at least 7 days in the 3 months before diagnosis. \\
\hline Underweight & $\mathrm{BMI}<18.5$, i.e. Weight $(\mathrm{kg}) /$ Height $\times 2(\mathrm{~m})<18.5$. \\
\hline Tobacco use & Ever smoked tobacco by self-report. \\
\hline Drinking history & Drinking more than one unit (21 g pure alcohol) per week by self-report. \\
\hline Chronic bronchitis & Chronic bronchitis history by self-report. \\
\hline $\begin{array}{l}\text { Average family annual } \\
\text { income per capita }\end{array}$ & $\begin{array}{l}\text { Average annual income per capita of urban family = RMB } 27000 \text { (USD 3970), average annual income } \\
\text { per capita of rural family = RMB } 8000 \text { (USD 1176), (USD } 1=\text { RMB 6.8). }\end{array}$ \\
\hline $\begin{array}{l}\text { Average family living area } \\
\text { per capita }\end{array}$ & Average of urban family $=29 \mathrm{~m}^{2}$, average of rural family $=31 \mathrm{~m}^{2}$ \\
\hline
\end{tabular}

and spot sputum) for sputum smear and culture. Patients with smear-positive and/or culture-positive sputum were diagnosed as bacteriologically positive TB. Patients with active PTB included those with bacteriologically positive sputum, and those diagnosed only by changes on their chest radiographs-known as clinically diagnosed PTB [6].

Each county in China, according to the national guideline [14], has a TB diagnosis group, composed of at least three health staff, including a clinical doctor, a laboratory technician and a radiologist. They are trained to diagnose active PTB cases based on patient symptoms and clinical history, radiographic findings, bacteriological results, and response to antibiotics. A national expert group reviewed the data from each patient involved in this study to confirm the diagnosis. Quality checks were done according to the national guidelines [14]. The survey process is shown in Fig. 1.

The data collected was reviewed by the enumerators carefully on the same day, and any mistakes were corrected within $24 \mathrm{~h}$. During the investigation period, $5 \%$ of questionnaires were randomly selected for review by supervisors. Meanwhile, the study invited the Westat Company (Rockville, Maryland, USA) as a third party to supervise the implementation process.

\section{Statistical analysis}

All data were double entered using an online input system developed by a local software company. TB prevalence of subgroups was respectively calculated, including bacteriologically positive $\mathrm{PTB}$ and active PTB. Chi-square $\left(\chi^{2}\right)$ test or Fisher's exact test was used to compare the difference of subgroups. Variables identified as potentially relevant for active PTB included: sex, age, residence, marital status, education level, family average annual income per capita, family living area per capita, tobacco use, drinking history, diabetes, close contacts, previous TB cases, and chronic bronchitis. Univariate logistic regression analysis was used to identify potential risk factors associated with active PTB. To control potential confounders, a multiple logistic regression model with stepwise selection was performed. All statistical tests were two-tailed, and the significance level was set at $P=0.05$ or less. Odds ratio $(O R)$ with $95 \%$ confidence interval $(C I)$ was calculated for categorical variables in the study to assess the strength of association between risk factors and TB prevalence. All tests were performed using SAS 9.3 (SAS Institute Inc., Cary, North Carolina, USA).

The population attributable fraction (PAF) is most commonly defined as the proportional reduction in average disease risk over a specified time interval that would be achieved by eliminating the exposure(s) of interest from the population while distributions of other risk factors in the population remain unchanged $[15,16]$. Formula as below was used to calculate PAF of each adjusted risk factor.

$$
P A F=\frac{p_{e}(R R-1)}{p_{e}(R R-1)+1}
$$

$p_{e}=$ proportion of source population exposed to the factor of interest. RR (risk ratio) may be the ratio of two cumulative incidence proportions, two (average) incidence rates (rate ratio), or an approximation of one of these ratios. In this study, $p_{e}$ means proportion of 


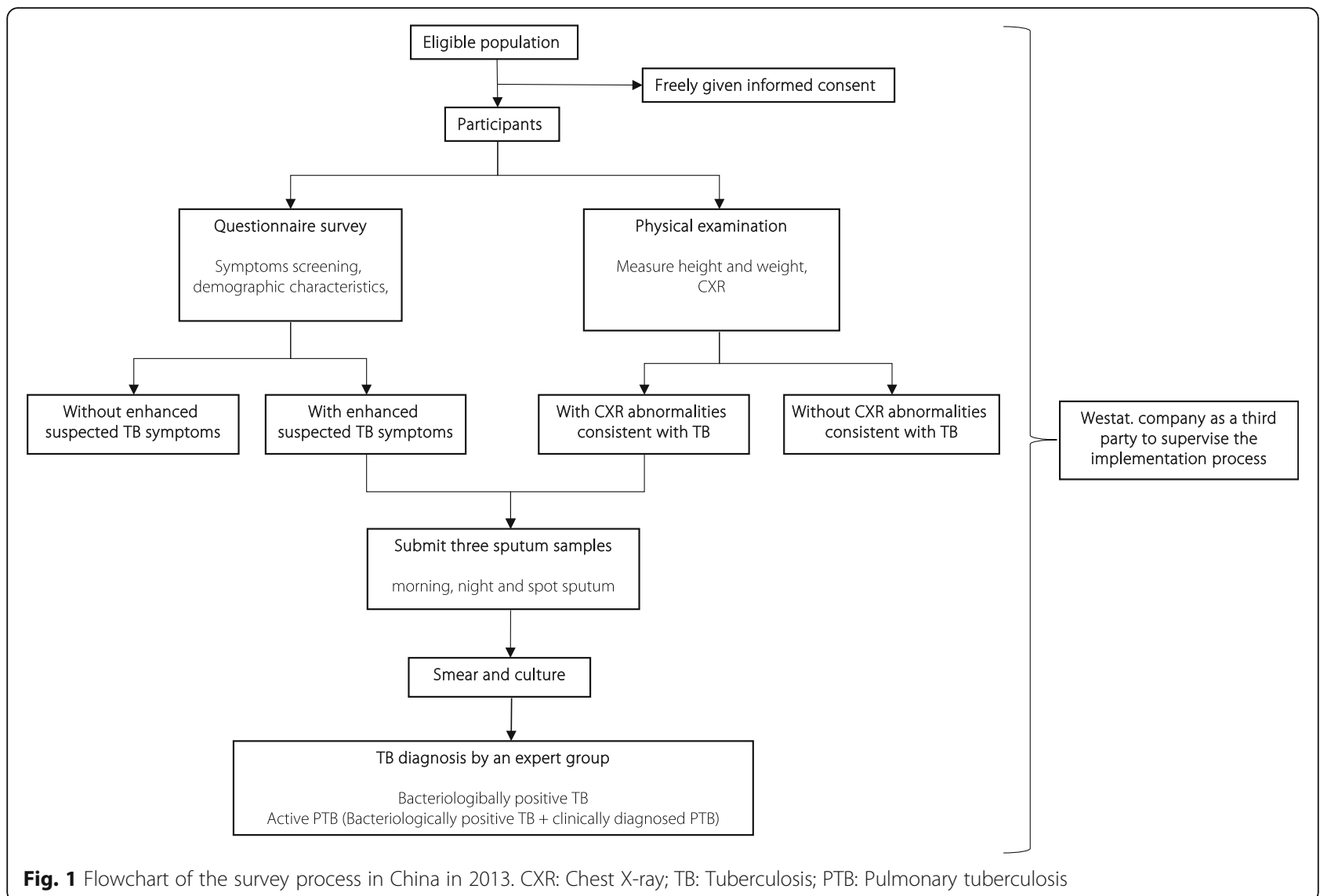

population exposed to adjusted risk factors, and $O R$ was used to replace RR.

\section{Ethical considerations}

The study was reviewed and approved by the Institutional Review Board of Chinese Center for Disease Control and Prevention. Written informed consent was signed by each participant before enrollment. All patients identified were referred to the local designated TB clinic or hospital for treatment according to national guidelines [14].

\section{Results}

\section{Demographic characteristics}

There were 38888 eligible elderly people $(\geq 65)$, of which 34269 (88.12\%) participants were included in this study and finished both the questionnaire and physical examination. Of these 18212 were male (53.14\%) and 16057 were female $(46.86 \%)$ (Table 2). The median age was 72 (interquartile range: 68-77).

Prevalence of bacteriologically positive TB and active PTB Among the 34269 participants, 817 (2.38\%) reported to have one or more of the TB symptoms identified, 2542 (7.42\%) had CXR abnormalities, and 168 (0.49\%) had both.
A total of 62 bacteriologically positive TB cases and 193 active PTB cases were identified. The prevalence of bacteriologically positive $\mathrm{PTB}$ and active $\mathrm{PTB}$ were $180.92 / 100000$, and $563.19 / 100000$ respectively. The prevalence of bacteriologically positive $\mathrm{TB}$ and active PTB were higher in males than in females, increased with age and were higher in rural over urban areas (Table 3).

\section{Multivariable logistic regression analysis for independent determinants of active PTB}

For active PTB, after adjustment in multivariable analysis, sex (M:F) $(O R=3.26$, 95\% CI: 2.34-4.55); using age group 65-74 as reference, age group 75-84 $(O R=1.59$, 95\% CI: $1.17-2.17)$, and age group $\geq 85(O R=2.05,95 \%$ $C I$ : $1.25-3.36)$; living in rural area $(O R=2.65,95 \% C I$ : 1.81-3.88); lower family income (OR: $1.64,95 \% C I$ : 1.17-2.31); using BMI group 18.5-23.9 as reference, BMI group $<18.5(O R=1.55,95 \% C I: 1.09-2.22)$, and BMI group $\geq 24$ (OR: 0.33, 95\% CI: 0.19-0.55); diabetes $(O R=1.83,95 \% C I: 1.08-3.10)$; close contacts $(O R=7.30,95 \% C I: 2.15-24.82)$; and previous TB cases $(O R=9.23, \quad 95 \% \quad C I: \quad 6.16-13.83)$, remained strongly associated (Table 4). 
Table 2 Demographic characteristics of the elderly participants aged 65 or over in China in 2013

\begin{tabular}{|c|c|c|}
\hline & Number & Proportion (\%) \\
\hline All & 34269 & 100.00 \\
\hline \multicolumn{3}{|l|}{ Sex } \\
\hline Female & 18212 & 53.14 \\
\hline Male & 16057 & 46.86 \\
\hline \multicolumn{3}{|l|}{ Age group } \\
\hline $65-74$ & 21685 & 63.28 \\
\hline $75-84$ & 10658 & 31.10 \\
\hline$\geq 85$ & 1926 & 5.62 \\
\hline \multicolumn{3}{|l|}{ Residence } \\
\hline Urban & 12933 & 37.74 \\
\hline Rural & 21336 & 62.26 \\
\hline \multicolumn{3}{|l|}{ Marital status } \\
\hline Married & 24953 & 72.82 \\
\hline Single/divorced & 8091 & 23.61 \\
\hline Unknown & 1225 & 3.57 \\
\hline \multicolumn{3}{|l|}{ Education level } \\
\hline Unknown & 1249 & 3.64 \\
\hline Illiterate or semi-illiterate ${ }^{a}$ & 12092 & 35.29 \\
\hline Elementary school & 12581 & 36.71 \\
\hline Secondary school & 7123 & 20.79 \\
\hline College and above & 1224 & 3.57 \\
\hline \multicolumn{3}{|c|}{ Family annual income per capita } \\
\hline Higher than average & 10854 & 31.67 \\
\hline Lower than average & 23415 & 68.33 \\
\hline \multicolumn{3}{|l|}{ Family living area per capita } \\
\hline Higher than average & 16434 & 47.96 \\
\hline Lower than average & 17835 & 52.04 \\
\hline
\end{tabular}

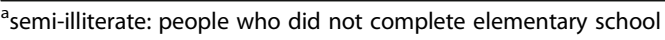

PAFs were considered for eight risk factors, as shown in Table 4. Male sex had the highest PAF (51.43\%), followed by living in a rural area (50.67\%), lower than average family annul income per capita (30.42\%), previous TB cases $(12.70 \%)$, underweight (5.52\%), diabetes (5.50\%), and close contacts $(1.70 \%)$. PAFs for age were $15.51 \%$ for $75-84$ years, and $5.57 \%$ for $\geq 85$ years.

\section{Discussion}

This study found that the TB prevalence of elderly people is very high in China, and confirmed that male sex, older age, living in rural areas, underweight, diabetes, close contact of PTB and previously TB history are high risk factors. The risk of $\mathrm{TB}$ increased with increasing age and decreasing BMI after adjusting for other factors, and there is a positive dose-response relationship.
Male sex is a strong risk factor for TB disease, supported by both this study and studies from high-income and low-income countries [2,17-19]. A study from the United States found that, among older adults aged 65 and above, TB rates increased with age [18], which was in accordance with our study. Residence in rural areas and with lower family incomes were risk factors in this study. However, this is not supported by studies from other low-income countries [3, 4, 20,21]. This may be because in China, the difference between living in urban versus rural areas are more significant than other Southeast Asian and African countries.

Our study showed TB risk decreased with increasing BMI, which is also found in two other studies [22, 23]. TB and diabetes mellitus comorbidity is universal globally, and older age is a risk factor for this comorbidity [24]. Jeon and Murray found the relative risk of diabetes was 3.11 (95\% CI: 2.27-4.26) [25], higher than this study. A systematic review by Morrison et al. of 41 studies showed that $4.5 \%$ of TB patient's household contacts were diagnosed with active TB [26], and another review by Shah et al. of 25 studies showed the pooled yield was 7.8\% (95\% CI: $5.6-10.0 \%)$ for active TB in household contacts of drug-resistant TB [27]. In our study, 3.2\% of TB patient's household contacts were diagnosed with active TB, and the OR was 7.30 (95\% CI: 2.15-24.82). In total, 5.4\% (33/ 193 ) of previous TB patients had a recurrence, with a high OR of 9.23 (95\% CI: 6.16-13.83) in this study. A retrospective cohort study conducted in Shanghai, China, showed 5.3\% (710/13417) of successfully treated cases had a recurrence, a rate of 7.55 (95\% CI: 7.01-8.13) episodes per 1000 person-years, more than 18 times the rate of TB in the general population [28]. More attention should be paid to patients with a history of previous TB diagnosis.

Heavy alcohol use and alcohol use disorders have a strong association with TB [29, 30], and this study showed the pooled relative risk was 2.94 (95\% CI: 1.89-4.59), which used an exposure cut-off level set at $40 \mathrm{~g}$ of alcohol per day or above, or defined exposure as a clinical diagnosis of an alcohol use disorder [29]. In our study, there was no association between alcohol use and TB, no matter if the alcohol use was prior to or during the study. We defined alcohol use as using $20 \mathrm{~g}$ alcohol per week or above, which was much lower than the above-mentioned study. This may have led to the difference. Systematic reviews also showed tobacco smoking and chronic respiratory disease were associated with an increased risk of TB [31, 32]. Our study found the TB prevalence was higher in tobacco smokers and patients with chronic bronchitis, but with no significant statistical difference.

Lönnroth et al. estimated PAFs of selected TB risk factors for 22 high-burden countries, and the PAFs of underweight and diabetes for the total Chinese population were 16.5 and $4.4 \%$ respectively [10]. The PAF of 
Table 3 TB Prevalence and comparison of subgroups of the elderly participants aged 65 or over in China in 2013

\begin{tabular}{|c|c|c|c|c|c|c|c|c|c|c|c|}
\hline \multirow[t]{2}{*}{ Category } & \multirow{2}{*}{$\begin{array}{l}\text { Number of } \\
\text { participants }\end{array}$} & \multicolumn{5}{|c|}{ Bacteriologically confirmed TB cases } & \multicolumn{5}{|c|}{ Active TB cases } \\
\hline & & Number & $\begin{array}{l}\text { Prevalence } \\
(95 \% \text { Cl) }\end{array}$ & $x^{2}$ & $P$ value & $\begin{array}{l}\text { Crude OR } \\
(95 \% \text { Cl) }\end{array}$ & Number & $\begin{array}{l}\text { Prevalence } \\
(95 \% \mathrm{Cl})\end{array}$ & $x^{2}$ & $P$ value & $\begin{array}{l}\text { Crude OR } \\
(95 \% \text { Cl) }\end{array}$ \\
\hline$\overline{\text { All }}$ & 34269 & 62 & $\begin{array}{l}180.92 \\
(135.89-225.96)\end{array}$ & & & & 193 & $\begin{array}{l}563.19 \\
(483.73-642.65)\end{array}$ & & & \\
\hline \multicolumn{12}{|l|}{ Sex } \\
\hline Female & 18212 & 17 & $\begin{array}{l}93.35 \\
(48.97-137.72)\end{array}$ & 16.51 & $<0.0001$ & ref & 49 & $\begin{array}{l}269.05 \\
(193.72-344.39)\end{array}$ & 60.05 & $<0.0001$ & ref \\
\hline Male & 16057 & 45 & $\begin{array}{l}280.25 \\
(198.37-362.14)\end{array}$ & & & $\begin{array}{l}3.00 \\
(1.72-5.26)\end{array}$ & 144 & $\begin{array}{l}896.81 \\
(750.33-1043.28)\end{array}$ & & & $\begin{array}{l}3.35 \\
(2.42-4.64)\end{array}$ \\
\hline \multicolumn{12}{|l|}{ Age(years) } \\
\hline $65-74$ & 21685 & 22 & $\begin{array}{l}101.45 \\
(59.06-143.85)\end{array}$ & 25.76 & $<0.0001$ & ref & 93 & $\begin{array}{l}428.87 \\
(341.70-516.03)\end{array}$ & 22.58 & $<0.0001$ & ref \\
\hline $75-84$ & 10658 & 30 & $\begin{array}{l}281.48 \\
(180.75-382.20)\end{array}$ & & & $\begin{array}{l}2.70 \\
(1.56-4.69)\end{array}$ & 79 & $\begin{array}{l}741.23 \\
(577.77-904.68)\end{array}$ & & & $\begin{array}{l}1.72 \\
(1.27-2.33)\end{array}$ \\
\hline$\geq 85$ & 1926 & 10 & $\begin{array}{l}519.21 \\
(197.40-841.02)\end{array}$ & & & $\begin{array}{l}3.93 \\
(1.81-8.55)\end{array}$ & 21 & $\begin{array}{l}1090.34 \\
(624.00-1556.69)\end{array}$ & & & $\begin{array}{l}2.12 \\
(1.30-3.44)\end{array}$ \\
\hline \multicolumn{12}{|l|}{ Residence } \\
\hline Urban & 12933 & 14 & $\begin{array}{l}108.25 \\
(51.55-164.96)\end{array}$ & 6.07 & 0.0137 & ref & 35 & $\begin{array}{l}270.63 \\
(180.97-360.28)\end{array}$ & 31.75 & $<0.0001$ & ref \\
\hline Rural & 21336 & 48 & $\begin{array}{l}224.97 \\
(161.33-288.62)\end{array}$ & & & $\begin{array}{l}2.08 \\
(1.15-3.78)\end{array}$ & 158 & $\begin{array}{l}740.53 \\
(625.06-856.00)\end{array}$ & & & $\begin{array}{l}2.75 \\
(1.91-3.97)\end{array}$ \\
\hline \multicolumn{12}{|l|}{ Marital status } \\
\hline Married & 24953 & 43 & $\begin{array}{l}172.32 \\
(120.82-223.83)\end{array}$ & 0.51 & 0.7769 & ref & 137 & $\begin{array}{l}549.03 \\
(457.09-640.97)\end{array}$ & 0.78 & 0.6777 & ref \\
\hline $\begin{array}{l}\text { Single/ } \\
\text { divorced }\end{array}$ & 8091 & 17 & $\begin{array}{l}210.11 \\
(110.23-309.99)\end{array}$ & & & $\begin{array}{l}1.21 \\
(0.69-2.12)\end{array}$ & 47 & $\begin{array}{l}580.89 \\
(414.82-746.97)\end{array}$ & & & $\begin{array}{l}1.05 \\
(0.75-1.46)\end{array}$ \\
\hline Unknown & 1225 & 2 & $\begin{array}{l}163.27 \\
(19.76-589.39)\end{array}$ & & & $\begin{array}{l}0.73 \\
(0.18-3.03)\end{array}$ & 9 & $\begin{array}{l}734.69 \\
(336.33-1394.29)\end{array}$ & & & $\begin{array}{l}1.03 \\
(0.53-2.03)\end{array}$ \\
\hline \multicolumn{12}{|l|}{ Education level } \\
\hline Unknown & 1249 & 2 & $\begin{array}{l}160.13 \\
(19.38-578.06)\end{array}$ & 5.89 & 0.2076 & - & 9 & $\begin{array}{l}720.58 \\
(329.86-1367.49)\end{array}$ & 9.64 & 0.0469 & $\begin{array}{l}6.41 \\
(0.81-50.58)\end{array}$ \\
\hline $\begin{array}{l}\text { Illiterate or } \\
\text { semi-illiterate }\end{array}$ & 12092 & 28 & $\begin{array}{l}231.56 \\
(145.79-317.33)\end{array}$ & & & - & 76 & $\begin{array}{l}628.51 \\
(487.21-769.82)\end{array}$ & & & $\begin{array}{l}6.84 \\
(0.95-49.16)\end{array}$ \\
\hline $\begin{array}{l}\text { Elementary } \\
\text { school }\end{array}$ & 12581 & 24 & $\begin{array}{l}190.76 \\
(114.44-267.09)\end{array}$ & & & - & 77 & $\begin{array}{l}612.03 \\
(475.33-748.74)\end{array}$ & & & $\begin{array}{l}6.99 \\
(0.97-50.21)\end{array}$ \\
\hline $\begin{array}{l}\text { Secondary } \\
\text { school }\end{array}$ & 7123 & 8 & $\begin{array}{l}112.31 \\
(48.43-221.26)\end{array}$ & & & - & 30 & $\begin{array}{l}421.17 \\
(270.46-571.88)\end{array}$ & & & $\begin{array}{l}4.84 \\
(0.66-35.48)\end{array}$ \\
\hline $\begin{array}{l}\text { College } \\
\text { and above }\end{array}$ & 1224 & 0 & $\begin{array}{l}0 \\
(0-301.47)\end{array}$ & & & ref & 1 & $\begin{array}{l}81.7 \\
(2.07-455.07)\end{array}$ & & & ref \\
\hline \multicolumn{12}{|c|}{ Family annul income per capita } \\
\hline $\begin{array}{l}\text { Higher than } \\
\text { average }\end{array}$ & 10854 & 10 & $\begin{array}{l}92.13 \\
(35.03-149.24)\end{array}$ & 6.93 & 0.0085 & ref & 44 & $\begin{array}{l}405.38 \\
(285.60-525.16)\end{array}$ & 7.50 & 0.0062 & ref \\
\hline $\begin{array}{l}\text { Lower than } \\
\text { average }\end{array}$ & 23415 & 52 & $\begin{array}{l}222.08 \\
(161.72-282.44)\end{array}$ & & & $\begin{array}{l}2.41 \\
(1.23-4.75)\end{array}$ & 149 & $\begin{array}{l}636.34 \\
(534.17-738.52)\end{array}$ & & & $\begin{array}{l}1.57 \\
(1.12-2.20)\end{array}$ \\
\hline \multicolumn{12}{|c|}{ Family living area per capita } \\
\hline $\begin{array}{l}\text { Higher than } \\
\text { average }\end{array}$ & 16434 & 26 & $\begin{array}{l}158.21 \\
(97.40-219.02)\end{array}$ & 0.90 & 0.3422 & ref & 77 & $\begin{array}{l}468.54 \\
(363.89-573.2)\end{array}$ & 5.05 & 0.0246 & ref \\
\hline $\begin{array}{l}\text { Lower than } \\
\text { average }\end{array}$ & 17835 & 36 & $\begin{array}{l}201.85 \\
(135.91-267.79)\end{array}$ & & & $\begin{array}{l}1.28 \\
(0.77-2.11)\end{array}$ & 116 & $\begin{array}{l}650.41 \\
(532.04-768.77)\end{array}$ & & & $\begin{array}{l}1.39 \\
(1.04-1.86)\end{array}$ \\
\hline
\end{tabular}


Table 3 TB Prevalence and comparison of subgroups of the elderly participants aged 65 or over in China in 2013 (Continued)

\begin{tabular}{|c|c|c|c|c|c|c|c|c|c|c|c|}
\hline \multirow[t]{2}{*}{ Category } & \multirow{2}{*}{$\begin{array}{l}\text { Number of } \\
\text { participants }\end{array}$} & \multicolumn{5}{|c|}{ Bacteriologically confirmed TB cases } & \multicolumn{5}{|c|}{ Active TB cases } \\
\hline & & Number & $\begin{array}{l}\text { Prevalence } \\
(95 \% \text { CD }\end{array}$ & $x^{2}$ & $P$ value & $\begin{array}{l}\text { Crude } O R \\
(95 \% \text { Cl } \\
\end{array}$ & Number & $\begin{array}{l}\text { Prevalence } \\
(95 \% \text { C) }\end{array}$ & $x^{2}$ & $P$ value & $\begin{array}{l}\text { Crude OR } \\
(95 \% \mathrm{Cl}) \\
\end{array}$ \\
\hline \multicolumn{12}{|l|}{$\overline{\text { Smoking history }{ }^{\mathrm{a}}}$} \\
\hline Never smoke & 27493 & 44 & $\begin{array}{l}160.04 \\
(112.75-207.33)\end{array}$ & 2.55 & 0.2788 & ref & 130 & $\begin{array}{l}472.85 \\
(391.56-554.13)\end{array}$ & 17.30 & 0.0002 & ref \\
\hline Prior smoker & 1663 & 4 & $\begin{array}{l}240.53 \\
(65.54-615.75)\end{array}$ & & & $\begin{array}{l}1.55 \\
(0.56-4.31)\end{array}$ & 13 & $\begin{array}{l}781.72 \\
(356.77-1206.67)\end{array}$ & & & $\begin{array}{l}1.71 \\
(0.96-3.02)\end{array}$ \\
\hline $\begin{array}{l}\text { Current } \\
\text { smoker }\end{array}$ & 5104 & 13 & $\begin{array}{l}254.7 \\
(116.24-393.16)\end{array}$ & & & $\begin{array}{l}1.63 \\
(0.88-3.04)\end{array}$ & 47 & $\begin{array}{l}920.85 \\
(657.58-1184.11)\end{array}$ & & & $\begin{array}{l}2.01 \\
(1.44-2.80)\end{array}$ \\
\hline \multicolumn{12}{|l|}{ Drinking history ${ }^{\mathrm{b}}$} \\
\hline Never drink & 27670 & 49 & $\begin{array}{l}177.09 \\
(127.50-226.67)\end{array}$ & 0.32 & 0.8533 & ref & 144 & $\begin{array}{l}520.42 \\
(435.42-605.42)\end{array}$ & 3.20 & 0.2017 & ref \\
\hline $\begin{array}{l}\text { Prior alcohol } \\
\text { user }\end{array}$ & 1234 & 3 & $\begin{array}{l}243.11 \\
(50.16-710.70)\end{array}$ & & & $\begin{array}{l}1.36 \\
(0.42-4.37)\end{array}$ & 9 & $\begin{array}{l}729.34 \\
(333.87-1384.12)\end{array}$ & & & $\begin{array}{l}1.39 \\
(0.71-2.73)\end{array}$ \\
\hline $\begin{array}{l}\text { Current } \\
\text { alcohol user }\end{array}$ & 5313 & 9 & $\begin{array}{l}169.4 \\
(77.55-321.48)\end{array}$ & & & $\begin{array}{l}0.97 \\
(0.48-1.98)\end{array}$ & 37 & $\begin{array}{l}696.41 \\
(472.01-920.8)\end{array}$ & & & $\begin{array}{l}1.36 \\
(0.95-1.96)\end{array}$ \\
\hline \multicolumn{12}{|l|}{ BMl } \\
\hline$<18.5$ & 3639 & 15 & $\begin{array}{l}412.2 \\
(203.60-620.8)\end{array}$ & 15.01 & 0.0005 & $\begin{array}{l}2.07 \\
(1.12-3.82)\end{array}$ & 47 & $\begin{array}{l}1291.56 \\
(922.31-1660.82)\end{array}$ & 63.49 & $<0.0001$ & $\begin{array}{l}1.91 \\
(1.35-2.69)\end{array}$ \\
\hline $18.5-23.9$ & 20884 & 38 & $\begin{array}{l}181.96 \\
(124.10-239.81)\end{array}$ & & & ref & 130 & $\begin{array}{l}622.49 \\
(515.48-729.49)\end{array}$ & & & ref \\
\hline$\geq 24$ & 9746 & 9 & $\begin{array}{l}92.35 \\
(42.27-175.25)\end{array}$ & & & $\begin{array}{l}0.52 \\
(0.25-1.08)\end{array}$ & 16 & $\begin{array}{l}164.17 \\
(83.73-244.61)\end{array}$ & & & $\begin{array}{l}0.27 \\
(0.16-0.46)\end{array}$ \\
\hline \multicolumn{12}{|l|}{ Diabetes } \\
\hline No & 31867 & 54 & $\begin{array}{l}169.45 \\
(124.26-214.65)\end{array}$ & 3.31 & 0.0688 & ref & 177 & $\begin{array}{l}555.43 \\
(473.61-637.26)\end{array}$ & 0.49 & 0.4846 & ref \\
\hline Yes & 2402 & 8 & $\begin{array}{l}333.06 \\
(143.63-656.12)\end{array}$ & & & $\begin{array}{l}1.97 \\
(0.94-4.14)\end{array}$ & 16 & $\begin{array}{l}666.11 \\
(339.72-992.51)\end{array}$ & & & $\begin{array}{l}1.20 \\
(0.72-2.01)\end{array}$ \\
\hline \multicolumn{12}{|l|}{ Close contacts } \\
\hline No & 34175 & 62 & $\begin{array}{l}181.42 \\
(136.26-226.58)\end{array}$ & & $1.0000^{d}$ & ref & 190 & $\begin{array}{l}555.96( \\
476.91-635.02)\end{array}$ & & $0.0162^{d}$ & ref \\
\hline Yes & 94 & 0 & $0(0-3925.53)$ & & & $\begin{array}{l}2.89 \\
(0.18-47.03)\end{array}$ & 3 & $\begin{array}{l}3191.49 \\
(658.51-9329.79)\end{array}$ & & & $\begin{array}{l}5.90 \\
(1.85-18.79)\end{array}$ \\
\hline \multicolumn{12}{|l|}{ Previous TB cases } \\
\hline No & 33663 & 50 & $\begin{array}{l}148.53 \\
(107.36-189.70)\end{array}$ & 110.59 & $<0.0001$ & ref & 160 & $\begin{array}{l}475.3 \\
(401.65-548.95)\end{array}$ & 262.59 & $<0.0001$ & ref \\
\hline Yes & 606 & 12 & $\begin{array}{l}1980.2 \\
(859.80-3100.60)\end{array}$ & & & $\begin{array}{l}13.58 \\
(7.20-25.63)\end{array}$ & 33 & $\begin{array}{l}5445.54 \\
(3587.57-7303.52)\end{array}$ & & & $\begin{array}{l}12.06 \\
(8.22-17.70)\end{array}$ \\
\hline \multicolumn{12}{|c|}{ Chronic bronchitis ${ }^{c}$} \\
\hline No & 32521 & 58 & $\begin{array}{l}178.35 \\
(132.45-224.25)\end{array}$ & & $0.7694^{d}$ & ref & 175 & $\begin{array}{l}538.11 \\
(458.39-617.84)\end{array}$ & 3.94 & 0.0473 & ref \\
\hline Yes & 1647 & 3 & $\begin{array}{l}182.15 \\
(37.58-532.48)\end{array}$ & & & $\begin{array}{l}0.98(0.31- \\
3.13)\end{array}$ & 15 & $\begin{array}{l}910.75 \\
(449.85-1371.65)\end{array}$ & & & $\begin{array}{l}0.59 \\
(0.35-1.00)\end{array}$ \\
\hline
\end{tabular}

BMI Body mass index, Cl Confidence interval, OR Odds ratio, PAF Population attributable fraction, $T B$ Tuberculosis

${ }^{\mathrm{a}} 9$ missing

$\mathrm{b}_{52}$ missing

${ }^{c} 101$ missing

${ }^{\mathrm{d}}$ Fisher's Exact Test

underweight in our study was different from that estimation, because of a lower exposure rate and different target population. A study in Zimbabwe had a similar PAF of male sex (40\%) and household contacts (10\%), but with a high PAF of HIV (33\%) [33]. Our study came to the same conclusion as a study in Tanzania [34], that a factor strongly associated with an outcome on an individual level, such as close contact and TB $(O R=7.30$, PAF $=1.70 \%$ ), may not have much population impact, if the exposure is not common in the population at large. 
Table 4 Multivariable logistic regression analysis of active PTB and PAF of high-risk factors among the elderly participants aged 65 or over in China in 2013

\begin{tabular}{|c|c|c|c|c|}
\hline Category & Number of participants & Proportion (\%) & Adjusted OR (95\% Cl) & PAF (\%) \\
\hline \multicolumn{5}{|l|}{ Sex } \\
\hline Female & 18212 & 53.14 & ref & - \\
\hline Male & 16057 & 46.86 & $3.26(2.34-4.55)$ & 51.43 \\
\hline \multicolumn{5}{|l|}{ Age(years) } \\
\hline $65-74$ & 21685 & 63.28 & ref & - \\
\hline $75-84$ & 10658 & 31.10 & $1.59(1.17-2.17)$ & 15.51 \\
\hline$\geq 85$ & 1926 & 5.62 & $2.05(1.25-3.36)$ & 5.57 \\
\hline \multicolumn{5}{|l|}{ Residence } \\
\hline Urban & 12933 & 37.74 & ref & - \\
\hline Rural & 21336 & 62.26 & $2.65(1.81-3.88)$ & 50.67 \\
\hline \multicolumn{5}{|c|}{ Family annul income per capita } \\
\hline Higher than average & 10854 & 31.67 & ref & - \\
\hline Lower than average & 23415 & 68.33 & $1.64(1.17-2.31)$ & 30.42 \\
\hline \multicolumn{5}{|l|}{ BMl } \\
\hline$<18.5$ & 3639 & 10.62 & $1.55(1.09-2.22)$ & 5.52 \\
\hline $18.5-23.9$ & 20884 & 60.94 & ref & - \\
\hline$\geq 24$ & 9746 & 28.44 & $0.33(0.19-0.55)$ & - \\
\hline \multicolumn{5}{|l|}{ Diabetes } \\
\hline No & 31867 & 92.99 & ref & - \\
\hline Yes & 2402 & 7.01 & $1.83(1.08-3.10)$ & 5.50 \\
\hline \multicolumn{5}{|l|}{ Close contacts } \\
\hline No & 34175 & 99.73 & ref & - \\
\hline Yes & 94 & 0.27 & $7.30(2.15-24.82)$ & 1.70 \\
\hline \multicolumn{5}{|l|}{ Previous TB cases } \\
\hline No & 33663 & 98.23 & ref & - \\
\hline Yes & 606 & 1.77 & $9.23(6.16-13.83)$ & 12.70 \\
\hline
\end{tabular}

BMI Body mass index, CI Confidence interval, OR Odds ratio, PAF Population attributable fraction, TB Tuberculosis, PTB Pulmonary tuberculosis

Dye and Williams thought that control programs had been less effective than expected in cutting transmission mainly because patients were not diagnosed and cured quickly enough [35]. To find more undiagnosed TB cases, ACF is an effective method. However, ACF can be a costly undertaking, depending on the target population and the diagnostic strategy used [36]. So, the first step to implement ACF is to confirm the target population with high enough prevalence. This study indicated that ACF could be implemented among elderly people aged 65 and above with low BMI $(<18.5)$, diabetes, close contact history and previous TB history as a priority, because of the higher risks and minor population number. The proportions of low BMI $(<18.5)$, diabetes, close contact history and previous TB history among elderly people are $10.62,7.01,0.27$ and $1.77 \%$ respectively. Conducting ACF among these high-risk groups of elderly people will be more efficient and cost-effective. For other high-risk factors with a large population number, like male sex or residence in rural areas, it will be not as easy to screen them all. However, high-risk factors combination will be a better choice, which can increase the risk of development of recent active $\mathrm{TB}$ and narrow the target population [37].

The strengths of this study were that it was a carefully designed and implemented survey, which used the current TB diagnostic protocols and tests in China for diagnosis. And the results may represent the prevalence and case distribution characteristics of TB among elderly people in China. The study also showed the yield of ACF. The study followed the Strengthening the Reporting of Observational Studies in Epidemiology (STROBE) guidelines [38] and sound ethics principles for the conduct and reporting of this study [39].

The study had a few limitations. Some TB risk factors were collected by self-reporting (such as tobacco use and drinking history) or based on self-reporting and local health documents (such as diabetes), which might 
not be sufficient to estimate the real distribution among the elderly people. The prevalence of diabetes in this study was $7 \%$, much lower than the $10.9 \%$ of the national diabetes prevalence survey in China in 2013 [40]. Meanwhile, our study was unable to detect any association between HIV and TB, as there was only one person who was living with HIV.

\section{Conclusions}

The TB prevalence of elderly people is very high in China, and male sex, older age, living in rural areas, underweight, diabetes, close contact of PTB and previous TB history are high risk factors. The risk of TB increased with increasing age and decreasing BMI after adjusting for other factors, and there is a positive dose-response relationship. ACF could be implemented among elderly people aged 65 and above with underweight, diabetes, close contact history and previous TB history as a priority, which will get significant yields and be cost-effective.

\section{Additional file}

Additional file 1: Multilingual abstracts in the five official working languages of the United Nations. (PDF $242 \mathrm{~kb}$ )

\section{Abbreviations}

ACF: Active case finding; AIDS: Acquired immune deficiency syndrome; BMI: Body mass index; Cl: Confidence interval; CXR: Chest X-ray; HIV: Human immunodeficiency virus; OR: Odds ratio; PAF: Population attributable fraction; PTB: Pulmonary tuberculosis; TB: Tuberculosis

\section{Acknowledgements}

We thank the tireless contributions of the staff in the provincial CDCs, local CDCs, and other related health care workers in undertaking the primary study. The study sites were located in Jiangsu Province, Zhejiang Province, Guangdong Province and Shanghai of eastern China, Henan Province, Heilongjiang Province and Hubei Province of central China, and Sichuan Province, Guangxi Zhuang Autonomous Region and Yunnan Province of western China.

\section{Funding}

The study was funded by The National Twelfth Five-year Mega-Scientific Projects of Infectious Diseases in China (Grant No.: 2013ZX10003004-001).

\section{Availability of data and materials}

The National Center for Tuberculosis Control and Prevention (NCTB) is the custodian of the data for this study. The data are not accessible online, but may be made available upon written request to the NCTB through the authors, if in line with the Ethical Review Board guidelines.

\section{Authors' contributions}

LW, HZ, and JC designed the study. CZ, FZ, YX oversaw data management. $\mathrm{CZ}, \mathrm{FZ}, \mathrm{HZ}$, and JC did data analysis and wrote the report. LW commented on the report and improved English writing. $Y Y, X S, W L, X W, J X, J Y, J L, F L$, $J W$, and LX organized investigations at the study sites. All authors contributed to review and revision and have seen and approved the final version of manuscript.

\section{Ethics approval and consent to participate}

The study was reviewed and approved by the Institutional Review Board of Chinese Center for Disease Control and Prevention. Written informed consent was signed by each participant before enrollment. All patients identified were referred to the local designated TB clinic or hospital for treatment according to China National TB Control Guideline.
Consent for publication

We got all co-authors' consent for publication.

\section{Competing interests}

The authors declare that they have no competing interests.

\section{Author details}

${ }^{1}$ National Center for Tuberculosis Control and Prevention, Chinese Center for Disease Control and Prevention, Beijing, China. ${ }^{2}$ Heilongjiang Provincial Center for Tuberculosis Control and Prevention, Harbin, Heilongjiang, China. ${ }^{3}$ Shanghai Municipal Center for Disease Control and Prevention, Shanghai, China. ${ }^{4}$ Jiangsu Provincial Center for Disease Control and Prevention, Nanjing, Jiangsu, China. ${ }^{5}$ Zhejiang Provincial Center for Disease Control and Prevention, Hangzhou, Zhejiang, China. ${ }^{6}$ Henan Provincial Center for Disease Control and Prevention, Zhengzhou, Henan, China. ${ }^{7}$ Hubei Provincial Center for Disease Control and Prevention, Wuhan, Hubei, China. ${ }^{8}$ Center for Tuberculosis Control of Guangdong Province, Guangzhou, Guangdong, China. ${ }^{9}$ Guangxi Provincial Center for Disease Control and Prevention, Nanning, Guangxi, China. ${ }^{10}$ Sichuan Provincial Center for Disease Control and Prevention, Chengdu, Sichuan, China. ${ }^{11}$ Yunnan Provincial Center for Disease Control and Prevention, Kunming, Yunnan, China.

Received: 19 June 2018 Accepted: 1 January 2019

Published online: 18 January 2019

\section{References}

1. National Bureau of Statistics of China. Statistical Communiqué of the People's Republic of China on the 2016 National Economic and Social Development: National Bureau of Statistics of China. Available from: http:// www.stats.gov.cn/english/PressRelease/201702/t20170228_1467503.html. Accessed 17 Oct 2017

2. Chadha VK, Kumar P, Anjinappa SM, Singh S, Narasimhaiah S, Joshi MV, et al Prevalence of pulmonary tuberculosis among adults in a rural sub-district of South India. PLoS One. 2012;7(8):e42625. https://doi.org/10.1371/journal. pone.0042625.

3. Hoa NB, Sy DN, Nhung NV, Tiemersma EW, Borgdorff MW, Cobelens FG. National survey of tuberculosis prevalence in Vietnam. Bull World Health Organ. 2010;88(4):273-80. https://doi.org/10.2471/BLT.09.067801.

4. Law I, Sylavanh P, Bounmala S, Nzabintwali F, Paboriboune P, lem V, et al. The first national tuberculosis prevalence survey of Lao PDR (2010-2011). Trop Med Int Health. 2015;20(9):1146-54. https://doi.org/10.1111/tmi.12536.

5. Rao VG, Bhat J, Yadav R, Gopalan GP, Nagamiah S, Bhondeley MK, et al. Prevalence of pulmonary tuberculosis--a baseline survey in central India. PLoS One. 2012;7(8):e43225. https://doi.org/10.1371/journal.pone.0043225.

6. Wang L, Zhang H, Ruan Y, Chin DP, Xia Y, Cheng S, et al. Tuberculosis prevalence in China, 1990-2010; a longitudinal analysis of national survey data. Lancet. 2014;383(9934):2057-64. https://doi.org/10.1016/S01406736(13)62639-2.

7. Wang $Y$, Wang W, Liu X, Wang L. Data compilation of the fifth national tuberculosis epidemiological sampling survey. Beijing: Military Medical Science Publishing House; 2011. p. 31-2.

8. Bele $S$, Jiang $W, L u H$, You H, Fan H, Huang L, et al. Population aging and migrant workers: bottlenecks in tuberculosis control in rural China. PLoS One. 2014;9(2):e88290. https://doi.org/10.1371/journal.pone.0088290.

9. Golub JE, Mohan Cl, Comstock GW, Chaisson RE. Active case finding of tuberculosis: historical perspective and future prospects. Int J Tuberc Lung Dis. 2005:9(11):1183-203.

10. Lönnroth K, Castro KG, Chakaya JM, Chauhan LS, Floyd K, Glaziou P, et al. Tuberculosis control and elimination 2010-50: cure, care, and social development. Lancet. 2010;375(9728):1814-29. https://doi.org/10.1016/ S0140-6736(10)60483-7.

11. Uplekar M, Weil D, Lonnroth $K$, Jaramillo E, Lienhardt C, Dias HM, et al. WHO's new end TB strategy. Lancet. 2015;385(9979):1799-801. https://doi. org/10.1016/S0140-6736(15)60570-0.

12. World Health Organization. Global Tuberculosis Report 2018. Available from: http://apps.who.int/iris/bitstream/handle/10665/274453/9789241565646-eng. pdf?ua=1. Accessed 27 Oct 2018 .

13. National Health Commission of the People's Republic of China. Criteria of weight for adults: National Health Commission of the People's Republic of China. Available from: http://www.nhfpc.gov.cn/ewebeditor/uploadfile/ 2013/08/20130808135715967.pdf. Accessed 20 Aug 2018 
14. Xiao D, Zhao M, Wang Y, Wang L, Xu S, Wang W. Guidelines for implementing the national tuberculosis control program in China (2008). Beijing: Union Medical College Press; 2009. p. 12-50.

15. Rockhill B, Newman B, Weinberg C. Use and misuse of population attributable fractions. Am J Public Health. 1998;88(1):15-9.

16. Poole $\mathrm{C}$. A history of the population attributable fraction and related measures. Ann Epidemiol. 2015;25(3):147-54. https://doi.org/10.1016/j. annepidem.2014.11.015.

17. Horton KC, MacPherson P, Houben RMGJ, White RG, Corbett EL. Sex differences in tuberculosis burden and notifications in low- and middleincome countries: a systematic review and meta-analysis. PLoS Med. 2016; 13(9):e1002119. https://doi.org/10.1371/journal.pmed.1002119.

18. Hochberg NS, Horsburgh CR. Prevention of tuberculosis in older adults in the United States: obstacles and opportunities. Clin Infect Dis. 2013;56(9): 1240-7. https://doi.org/10.1093/cid/cit027.

19. Onozaki I, Law I, Sismanidis C, Zignol M, Glaziou P, Floyd K. National tuberculosis prevalence surveys in Asia, 1990-2012: an overview of results and lessons learned. Trop Med Int Heal. 2015;20(9):1128-45. https://doi.org/ 10.1111/tmi.12534.

20. Zaman K, Hossain S, Banu S, Quaiyum MA, Barua PC, Salim MAH, et al. Prevalence of smear-positive tuberculosis in persons aged $\geqslant 15$ years in Bangladesh: results from a national survey, 2007-2009. Epidemiol Infect. 2012;140(6):1018-27. https://doi.org/10.1017/S0950268811001609.

21. Siroka A, Law I, Macinko J, Floyd K, Banda RP, Hoa NB, et al. The effect of household poverty on tuberculosis. Int J Tuberc Lung Dis. 2016;20(12):1603-8.

22. Lönnroth K, Williams BG, Cegielski P, Dye C. A consistent log-linear relationship between tuberculosis incidence and body mass index. Int J Epidemiol. 2010;39(1):149-55. https://doi.org/10.1093/ije/dyp308.

23. Tverdal A. Body mass index and incidence of tuberculosis. Eur J Respir Dis. 1986:69(5):355-62.

24. Workneh MH, Bjune GA, Yimer SA. Prevalence and associated factors of tuberculosis and diabetes mellitus comorbidity: a systematic review. PLoS One. 2017;12(4):e0175925. https://doi.org/10.1371/journal.pone.0175925.

25. Jeon CY, Murray MB. Diabetes mellitus increases the risk of active tuberculosis: a systematic review of 13 observational studies. PLoS Med. 2008;5(7):e152. https://doi.org/10.1371/journal.pmed.0050152.

26. Morrison J, Pai M, Hopewell PC. Tuberculosis and latent tuberculosis infection in close contacts of people with pulmonary tuberculosis in low-income and middle-income countries: a systematic review and meta-analysis. Lancet Infect Dis. 2008:8(6):359-68. https://doi.org/10.1016/S1473-3099(08)70071-9.

27. Shah NS, Yuen CM, Heo M, Tolman AW, Becerra MC. Yield of contact investigations in households of patients with drug-resistant tuberculosis: systematic review and meta-analysis. Clin Infect Dis. 2014;58(3):381-91. https://doi.org/10.1093/cid/cit643.

28. Shen X, Yang C, Wu J, Lin S, Gao X, Wu Z, et al. Recurrent tuberculosis in an urban area in China: relapse or exogenous reinfection? Tuberculosis. 2017; 103:97-104. https://doi.org/10.1016/j.tube.2017.01.007.

29. Lönnroth K, Williams BG, Stadlin S, Jaramillo E, Dye C. Alcohol use as a risk factor for tuberculosis - a systematic review. BMC Public Health. 2008;8:289. https://doi.org/10.1186/1471-2458-8-289.

30. Rehm J, Samokhvalov AV, Neuman MG, Room R, Parry C, Lönnroth K, et al. The association between alcohol use, alcohol use disorders and tuberculosis (TB). A systematic review. BMC Public Health. 2009:9:450. https://doi.org/10. 1186/1471-2458-9-450

31. Slama K, Chiang CY, Enarson DA, Hassmiller K, Fanning A, Gupta P, et al. Tobacco and tuberculosis: a qualitative systematic review and meta-analysis. Int J Tuberc Lung Dis. 2007;11(10):1049-61.

32. Lin H-H, Ezzati M, Murray M. Tobacco smoke, indoor air pollution and tuberculosis: a systematic review and meta-analysis. PLoS Med. 2007; 4(1):e20.

33. Corbett EL, Bandason T, Cheung YB, Makamure B, Dauya E, Munyati SS, et al. Prevalent infectious tuberculosis in Harare, Zimbabwe: burden, risk factors and implications for control. Int J Tuberc Lung Dis. 2009;13(10): $1231-7$.

34. Senkoro M, Kumar AMV, Chinnakali P, Mfinanga SG, Egwaga S, Kamara V, et al. Population impact of factors associated with prevalent pulmonary tuberculosis in Tanzania. Int J Tuberc Lung Dis. 2016;20(10):1326-33.

35. Dye C, Williams BG. The population dynamics and control of tuberculosis. Science. 2010;328(5980):856-61. https://doi.org/10.1126/science.1185449.

36. Nishikiori N, Van Weezenbeek C. Target prioritization and strategy selection for active case-finding of pulmonary tuberculosis: a tool to support country-level project planning. BMC Public Health. 2013;13:97. https://doi.org/10.1186/14712458-13-97.

37. Patra J, Jha P, Rehm J, Suraweera W. Tobacco smoking, alcohol drinking, diabetes, low body mass index and the risk of self-reported symptoms of active tuberculosis: individual participant data (IPD) meta-analyses of 72,684 individuals in 14 high tuberculosis burden countries. PLoS One. 2014;9(5): e96433. https://doi.org/10.1371/journal.pone.0096433.

38. von Elm E, Altman DG, Egger M, Pocock SJ, Gøtzsche PC, Vandenbroucke $J P$. The strengthening the reporting of observational studies in epidemiology (STROBE) statement: guidelines for reporting observational studies. Lancet. 2007;370(9596):1453-7.

39. Edginton M, Enarson D, Zachariah R, Reid T, Satyanarayana S, Bissell K, et al. Why ethics is indispensable for good-quality operational research. Public Health Action. 2012;2(1):21-2. https://doi.org/10.5588/pha.12.0001.

40. Wang L, Gao P, Zhang M, Huang Z, Zhang D, Deng Q, et al. Prevalence and ethnic pattern of diabetes and prediabetes in China in 2013. JAMA. 2017; 317(24):2515-23. https://doi.org/10.1001/jama.2017.7596.
Ready to submit your research? Choose BMC and benefit from:

- fast, convenient online submission

- thorough peer review by experienced researchers in your field

- rapid publication on acceptance

- support for research data, including large and complex data types

- gold Open Access which fosters wider collaboration and increased citations

- maximum visibility for your research: over $100 \mathrm{M}$ website views per year

At BMC, research is always in progress.

Learn more biomedcentral.com/submissions 